\title{
The Orbit and Optic Nerves
}

Magnetic resonance imaging (MRI) of the orbits and optic nerves has developed into the "gold standard" of imaging modalities for the evaluation of many soft tissue abnormalities of the orbit (see Mafee et al., 1996). Its advantage is a result of its superior soft tissue contrast, which allows for the excellent evaluation of fat, muscle, nervous tissue, cerebrospinal fluid, and blood vessels. Computed tomography (CT) remains the modality of choice for evaluation of the bony structures of the orbit. MRI is also more flexible, allowing for multiplanar imaging that is not possible with CT. For these reasons, CT and MRI can be complementary, especially in situations where one encounters entities such as sinus pathology, which bridges the air spaces, bone, orbit, and brain. Ultrasound also has many advantages for evaluation of the globes, but it does not provide the more universal views of the orbit and periorbital area that are possible with MRI.

\section{IMAGING OF THE ORBITS AND BRAIN}

In most situations it is important not to focus strictly on the orbits, but to also image the adjacent structures, including the face, scalp, brain, sinuses, and blood vessels-in particular the cavernous sinus. Trying to determine the location of pathology based on clinical symptoms alone is frequently not very accurate, even after a detailed clinical exam. For example, a patient may present with diplopia, which suggests an extraocular muscle disorder, but the true etiology may be a dural arteriovenous fistula of the posterior fossa, with secondary venous congestion of the cavernous sinus. In a case where the pathology is not where it is anticipated to exist, focusing the exam exclusively on the orbits would overlook the true underlying pathology. There are a few indications where a very focused, detailed exam of the orbit is indicated, in isolation from other structures. This most commonly occurs when there is a question of a primary disorder of the globe, such as choroidal melanoma. Even in this case, however, it may be necessary to image the brain, in order to exclude and characterize more distant metastatic disease.

A standard head coil (Table A7.5.1) is usually utilized for a general orbital examination, which allows for a fast and simple setup. The exam is similar to that used for standard imaging of the head, but the focus of the exam is on the orbit and some of the pulse sequences used for the orbital exam are not routinely used for brain imaging. Table A7.5.1 lists the hardware necessary to perform the procedure, along with appropriate parameters.

Table A7.5.1 Equipment Parameters for Orbit Imaging

\begin{tabular}{ll}
\hline Coil type & $\begin{array}{l}\text { Head coil or small diameter }(10 \mathrm{~cm}), \\
\text { circular, phased array surface coil } \\
\text { Fat-saturation pulse }\end{array}$ \\
Preferable \\
Cardiac gating & $20 \mathrm{mT} / \mathrm{m}$ \\
Peripheral gating & No \\
Respiratory gating & No \\
Respirator & No \\
Oxygen & No \\
Motion cushions & No \\
Use of contrast agents & Helpful \\
Susceptibility-reducing eye pads & Yes \\
\hline
\end{tabular}

BASIC

PROTOCOL 


\section{Materials}

Normal saline $(0.9 \% \mathrm{NaCl})$, sterile

Extravascular contrast agent (e.g., Magnevist, Omniscan, or Prohance)

\section{Set up equipment and patient}

1. Interview (screen) the patient to ensure that he or she has no contraindications such as cardiac pacemakers or other implants containing ferromagnetic materials. Also be sure to find out if the patient has any health conditions that may require the presence of special emergency equipment during the scanning procedure, or necessitate any other precautions.

In the case of an orbital exam, it is particularly important to confirm that the patient does not have metallic fragments or implants in the eyes (see Kelly et al., 1986; Lagouros et al., 1987; Otto et al., 1992). If there is a history of a possible metallic foreign body, then plain radiographs are recommended as an initial study. Metallic foreign bodies in the orbit are considered an absolute contraindication to MR, as there have been reports of blindness as a result. Although CT is more sensitive in detecting smaller metallic foreign bodies, and is advocated by some, plain films are adequate. There are a few metal implants now used in the eye, particularly with cataract surgery.

Generally standard screening forms are used for all patients scanned in a magnetic resonance system.

The presence of any ferromagnetic metals may be a health hazard to the patient when he or she is inside the magnet, and will also affect the imaging. If in doubt as to the exact composition of the items, it is best to exclude patients with any metal implants; see Shellock (1996) for discussion of what implants may be safely scanned using magnetic resonance.

Patients may be accompanied into the magnet room by a friend or family member, who can sit in the room during the scan and comfort the patient as needed. This companion must be screened as well to ensure the absence of loose metal objects on the body or clothing.

2. If the procedure is a research protocol, have the patient sign any necessary consent form.

3. Have the patient remove all jewelry and change into a gown to eliminate any metal that might be found in clothing.

One should double-check for any metallic objects.

4. Have the patient wash off any mascara and other makeup to avoid local tissue heating and image artifacts.

Mascara and other makeup commonly produce magnetic susceptibility artifacts (Fig. A7.5.1). This is particularly important in the evaluation of the orbit. Some tattoos will also produce artifacts (Sacco et al., 1987; Herrick et al., 1997) and possible heating.

5. Inform the patient about what will occur during the procedure, what he or she will experience while in the magnet, and how to behave, including the following:

a. If earphones or headphones are used to protect the ears from the loud sounds produced by the gradients, the patient will be asked to wear these, but will be able to communicate with you at any time during the imaging. Many systems have handsets that can be used to alert the technologists if there is a problem.

If the patient does not utilize a headset then earplugs are necessary.

b. The patient will be given a safety squeeze-bulb or similar equipment to request assistance at any time (demonstrate how this works).

c. For good results the patient should not talk, and should avoid or minimize swallowing or other movement, during each scan-i.e., as long as the banging sounds continue. Between scans, talking and swallowing are allowed in most 
cases, but should be avoided when comparative positional studies are being performed; the patient will be informed when this is the case.

d. Nevertheless, the patient may call out at any time if he or she feels it necessary.

6. Help the patient mount onto the table. Either before or right after the patient lies down, set up any triggering devices or other monitoring equipment that is to be used.

7. Instruct the patient to try to avoid scanning with their eyes throughout the procedure because this movement will lead to motion artifacts.

Since it is essentially impossible to have the patient fix their vision point for a long period of time, the best advice to give them is to keep their eyes closed. If the patient sleeps, there may be rapid eye movements that can cause artifacts (Fig. A7.5.2).

A

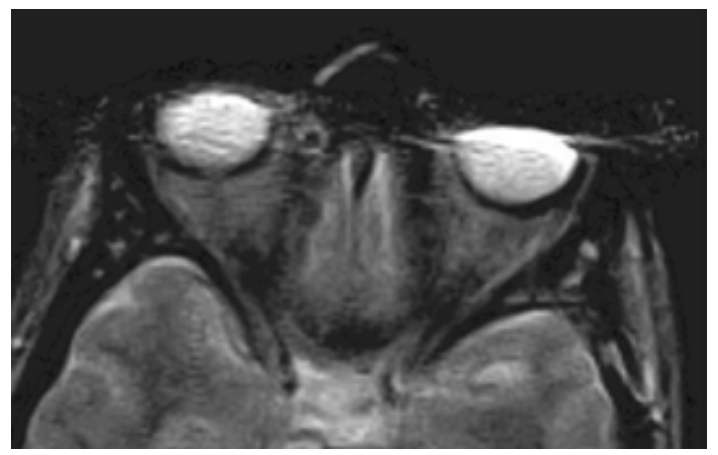

B

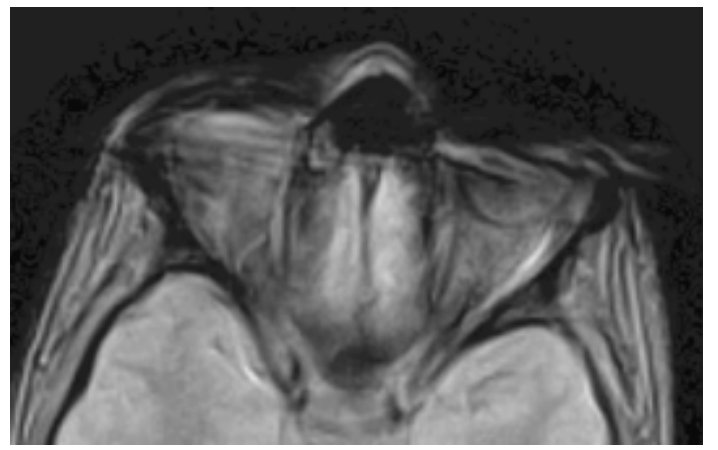

Figure A7.5.1 Magnetic susceptibility artifacts. Image (A) is a transverse $T_{2}$-weighted image. Image (B) is a transverse proton density image. Both images demonstrate magnetic susceptibility artifact from mascara distorting the globes. There is loss of signal and "smearing" of the image.

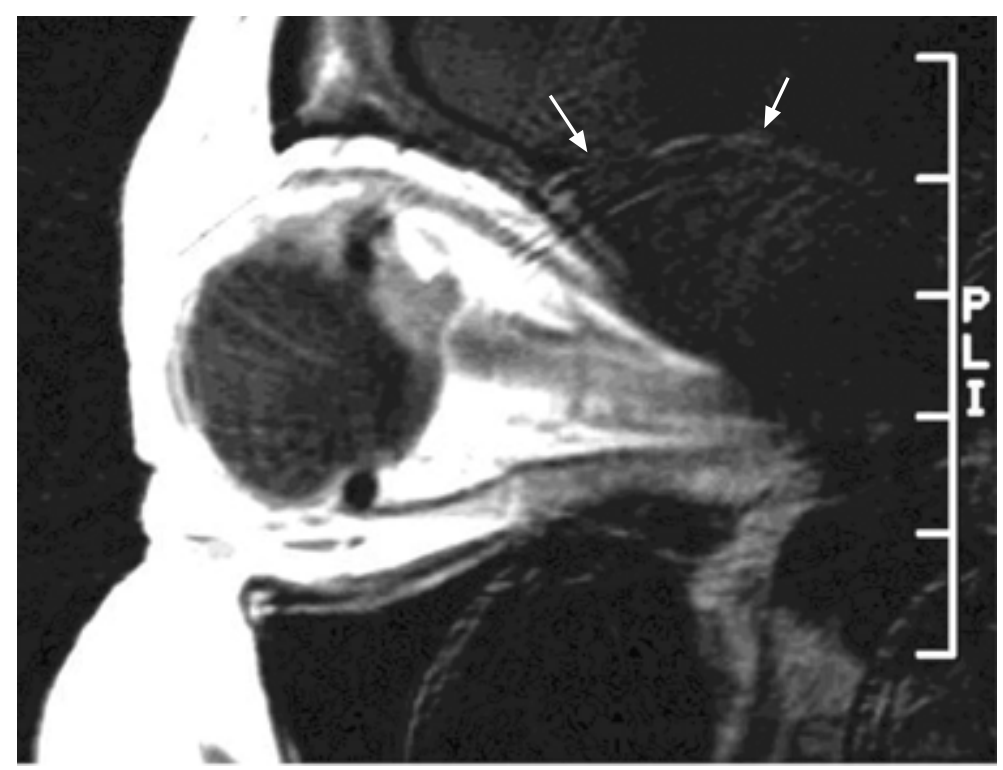

Figure A7.5.2 Sagittal $T_{1}$-weighted image phase encoded artifacts (ghosting). This is a sagittal image of the orbit demonstrating a choroidal melanoma. Note the phase encoding artifact (arrows) of the muscle cone. Since the globe cannot be fixed, motion artifacts are common. This patient demonstrates a sclera band and an invasive melanoma of the choroids along the superior posterior portion of the globe. 
For orbital imaging, it is best not to use visual stimuli because the stimuli will encourage eye motion.

8. Center the head in the head coil or adjust the surface coil.

It may be important to place sponges around the patient to help support the head in a comfortable position and to suppress motion during the scanning process. If the patient is not in a comfortable position he or she will most likely move during the exam.

9. If needed, place a pillow or other support under the knees to make the patient more comfortable.

10. Use the centering light to position the patient's nose and put him or her into the center of the magnet.

Once this step has been performed, so long as the patient does not move on the table, the table itself can be moved and then replaced in the same position as before without jeopardizing the positioning of one scan relative to another.

11. If the patient is unable to hold still, provide an appropriate sedative.

\section{Sequence 1: Sagittal $T_{1}$-weighted series}

12. Run $T_{1}$-weighted, short $T_{\mathrm{E}}$, short $T_{\mathrm{R}}$, spin-echo sequence (Table A7.5.2).

The field of view must be large $(24 \mathrm{~cm})$ so that portions of the head, and particularly the orbits, are not excluded from the image. In general, there is no great advantage to making the field of view very small, since wrap-around (aliasing) artifacts will degrade the image quality when utilizing a head coil. This sequence allows for good visualization of the fat spaces in the orbits and the resulting image obtained can be used as a planning image for the other sequences to follow.

\section{Sequence 2: $T_{2}$-weighted imaging}

13. Run transverse $T_{2}$-weighted imaging (sequence 2; Table A7.5.3).

Using the images generated in sequence 1, transverse images are planned to include most of the sinuses and brain. Set the imaging parameters as shown in Table A7.5.3. This sequence will make abnormalities with high water content and long $T_{2}$ more conspicuous, a finding that is of value in characterizing the vitreous and the sinuses. Since the images are quite $T_{2}$-weighted, the fat structures are partially suppressed.

Table A7.5.2 Parameters for Sagittal $T_{1}$-Weighted Images (Sequence 1)

\begin{tabular}{ll}
\hline Patient position & Supine \\
Scan type & Spin echo \\
Imaging plane (orientation) & Sagittal \\
Central slice or volume center & Laser light centered on nose \\
Echo time $\left(T_{\mathrm{E}}\right)$ & As short as possible \\
Repeat time $\left(T_{\mathrm{R}}\right)$ & $500 \mathrm{msec}$ \\
Flip angle $(\mathrm{FA})$ & $90^{\circ}$ \\
Fields of view $\left(\mathrm{FOV}, \mathrm{x}, \mathrm{FOV}_{\mathrm{y}}\right)$ & $240 \mathrm{~mm}, 240 \mathrm{~mm}$ \\
Resolution $(\Delta x, \Delta y)$ & $0.94 \mathrm{~mm}, 0.94 \mathrm{~mm}$ \\
Number of data points collected $\left(N_{\mathrm{x}}, N_{\mathrm{y}}\right)$ & 256,256 \\
Display matrix $\left(D_{\mathrm{x}}, D_{\mathrm{y}}\right)$ & 256,256 \\
Slice thickness $(\Delta z)$ & $3-5 \mathrm{~mm}$ \\
Number of slices & 15 \\
Slice gap & $1-2 \mathrm{~mm}$ \\
Number of acquisitions $\left(N_{\mathrm{acq}}\right)$ & $1-2$ \\
Swap read and phase encoding & Not applicable \\
Saturation pulses & Not applicable \\
Scan time & $2-4$ min \\
\hline
\end{tabular}


Table A7.5.3 Parameters for Transverse $T_{2}$-Weighted Images (Sequence 2)

\begin{tabular}{ll}
\hline Patient position & Supine \\
Scan type & Spin echo \\
Imaging plane (orientation) & Transverse \\
Central slice or volume center & Laser light centered on nose \\
Echo time $\left(T_{\mathrm{E}}\right)$ & $90 \mathrm{msec}$ \\
Repeat time $\left(T_{\mathrm{R}}\right)$ & $5000 \mathrm{msec}$ \\
Flip angle $(\mathrm{FA})$ & $90^{\circ}$ \\
Fields of view $(\mathrm{FOV}, \mathrm{FOV}$ & $\mathrm{y})$ \\
Resolution $(\Delta x, \Delta y)$ & $240 \mathrm{~mm}, 240 \mathrm{~mm}$ \\
Number of data points collected $\left(N_{\mathrm{x}}, N_{\mathrm{y}}\right)$ & $0.94 \mathrm{~mm}, 0.94 \mathrm{~mm}$ \\
Display matrix $\left(D_{\mathrm{x}}, D_{\mathrm{y}}\right)$ & 256,256 \\
Slice thickness $(\Delta z)$ & 256,256 \\
Number of slices & $3-5 \mathrm{~mm}$ \\
Slice gap & 20 \\
Number of acquisitions $\left(N_{\mathrm{acq}}\right)$ & $1-2 \mathrm{~mm}$ \\
Swap read and phase encoding & $1-2$ \\
Saturation pulses & Yes \\
Scan time & Not applicable \\
\hline
\end{tabular}

Short tau inversion recovery (STIR) coronal images can also be acquired to help suppress the orbital fat (Table 7.5.10). This sequence highlights the optic nerves. This type of sequence can help differentiate non-enhancing white matter lesions from contrast enhancement.

14. Let the patient know you are ready, and begin the scan. Remind the patient not to move the eyes.

\section{Sequence 3: High resolution transverse $T_{1}$-weighted contrast enhanced images}

15. Remove the patient from the scanner (the patient should not move on the table). Establish an intravenous line from which the contrast agent can be injected, and attach this line securely to the patient so that movement into or out of the magnet will not pull at the patient's arm. Move the patient back into the scanner.

It is preferable to insert the line prior to imaging and to leave the patient in the magnet, with no intervening motion between the scans run before contrast agent injection and those run after injection.

16. Leaving the patient in the magnet, inject the contrast agent, flush the i.v. line with 10 $\mathrm{ml}$ saline and run transverse $T_{1}$-weighted high-resolution scan (sequence 3; Table A7.5.4).

A dose of $0.1 \mathrm{mmol} / \mathrm{kg}$ of contrast agent is usually given.

Sequence 3 is a high-resolution $T_{1}$-weighted series that highlights the fat spaces of the bones and orbits (Fig. A7.5.3). The arterial blood vessels (carotid, ophthalmic, circle of Willis, and the internal maxillary branches) can all be seen as well. Small structures within the orbit, including some of the cranial nerves and the veins, can also be visualized as lower-signal-intensity regions.

Since these images are a three-dimensional acquisition and have high resolution of high quality, it is possible to post-process them into any other orientation plane or surface images.

If this type of high-resolution three-dimensional pulse sequence is not possible, then a proton-density or $T_{1}$-weighted spin-echo sequence with relatively thin slices would be an appropriate imaging alternative. 
Table A7.5.4 Parameters for Transverse $T_{1}$-Weighted High Resolution Images (Sequence 3)

\begin{tabular}{ll}
\hline Patient Position & Supine \\
Scan type & 3-dimensional gradient echo \\
Imaging plane (orientation) & Transverse \\
Central slice or volume center & Laser light centered on nose \\
Echo time $\left(T_{\mathrm{E}}\right)$ & As short as possible \\
Repeat time $\left(T_{\mathrm{R}}\right)$ & $50 \mathrm{msec}$ \\
Flip angle $(\mathrm{FA})$ & $30^{\circ}$ \\
Fields of view $(\mathrm{FOV}, \mathrm{x}, \mathrm{FOV}$ & $\mathrm{y})$ \\
Resolution $(\Delta x, \Delta y)$ & $240 \mathrm{~mm}, 240 \mathrm{~mm}$ \\
Number of data points collected $\left(N_{\mathrm{x}}, N_{\mathrm{y}}\right)$ & $0.94 \mathrm{~mm}, 0.94 \mathrm{~mm}$ \\
Display matrix $\left(D_{\mathrm{x}}, D_{\mathrm{y}}\right)$ & 256,256 \\
Slice thickness $(\Delta z)$ & 512,256 \\
Number of slices & $1.5-2 \mathrm{~mm}$ \\
Slice gap & $28-37$ \\
Number of acquisitions $\left(N_{\mathrm{acq}}\right)$ & $0 \mathrm{~mm}$ \\
Swap read and phase encoding & 1 \\
Saturation pulses & Yes \\
Scan time & Not applicable \\
\hline
\end{tabular}

Sequence 4: Transverse $T_{1}$-weighted contrast enhanced imaging with fat saturation

17. Obtain slices from the anterior margins of the orbit back into the posterior fossa (sequence 4; Table A7.5.5).

This sequence takes advantage of the combination of fat saturation and contrast enhancement (see Barakos et al., 1991; Anzai et al., 1992; Amano et al., 1997). Since there are many fatty spaces in and around the orbit, recognition of contrast enhancement on $T_{1}$-weighted images may not be possible without fat saturation, since the fat is already high in signal intensity. Fat saturation makes the contrast enhancement more conspicuous. This is particularly important when imaging infiltrative lesions of the orbit. Fat saturation also makes the normally enhancing structures such as the extraocular muscles stand out (Fig. A7.5.4).

It is essential to use extra pre-scanning in order to determine the correct frequency to saturate fat.

If there is anything causing severe degradation of the image quality, such as metal artifact from dental hardware, it is recommended that the imaging sequence be repeated without fat saturation in order to ensure a good quality exam.

\section{Sequence 5: Coronal $T_{1}$-weighted contrast enhanced imaging with fat saturation}

18. Run coronal $T_{1}$-weighted fat saturated contrast enhanced sequence (Fig. A7.5.5) to evaluate the superior-inferior extension of pathology (sequence 5; Table A7.5.6).

Coronal imaging is also advantageous when evaluating the optic nerve and the extra-ocular muscles, since they are sectioned perpendicular to their courses. The transverse images have more problems with partial volume averaging and with the structures looping in and out of the section plane. Visualization of the cranial nerves in the cavernous sinus is possible, and it is also important to identify the carotid arteries. 


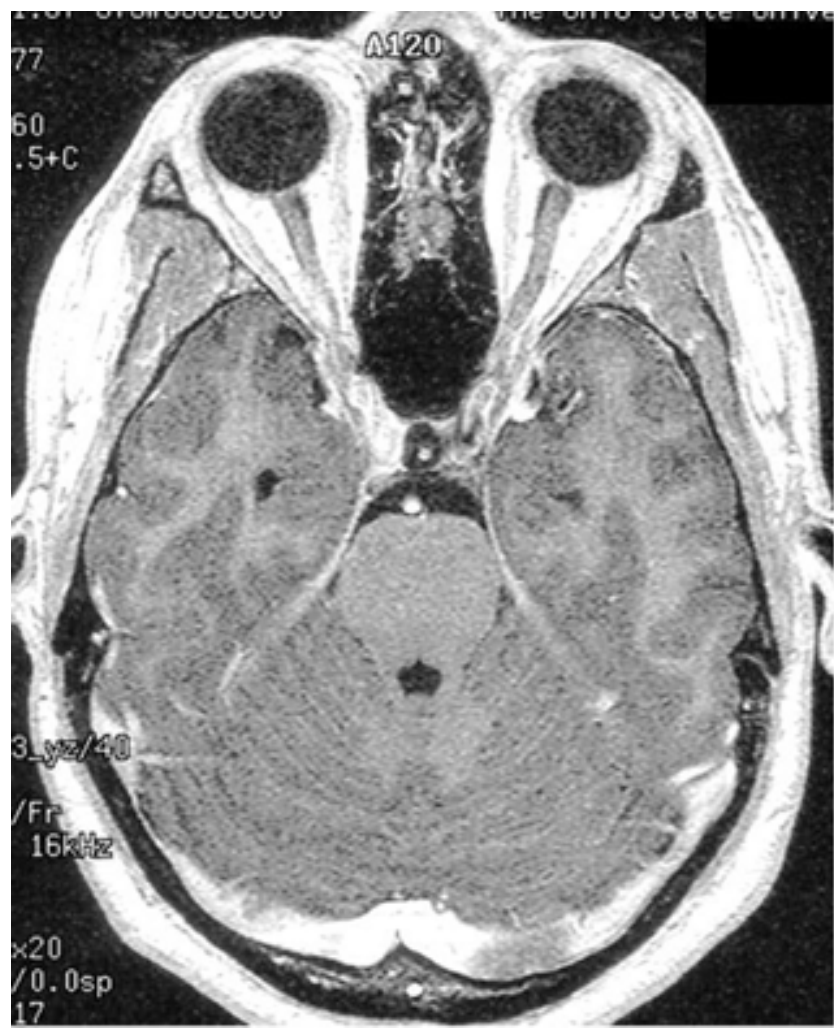

Figure A7.5.3 High-resolution transverse $T_{1}$-weighted enhanced image. This is a 3-D sequence and can be reformatted in any orientation since the image thickness is only 1.5 $\mathrm{mm}$ and the matrix is 512 . This sequence is used to create high-quality-resolution images. The higher the initial quality of the image, the better the reconstruction. This sequence is optimal for evaluation of cranial nerves intracranially, prior to their entering the orbit. The image is similar to a $T_{1}$-weighted spin echo sequence, but much higher resolution. Note the good definition of the gray and white matter, the cerebrospinal fluid (CSF) spaces, and the blood vessels.

\section{DEDICATED HIGH-RESOLUTION SURFACE COIL ORBITAL STUDY}

If the goal of the examination is to completely focus on the orbital contents, then the use of a high-resolution surface coil can be in order (see Atlas et al., 1987). Dedicated surface coils for the orbits are available, but more general-purpose coils used for small body parts such as the wrist and temporal mandibular joint (TMJ) can be used. A higher signal-tonoise ratio is possible with phased array coils. Because the sequences used for this type of exam are of very high resolution, unless the advantage of the surface coils (in terms of signal to noise) is greater than the loss of signal resulting from the high resolution and small field of view, this type of evaluation is not advantageous. The procedure below is most useful on high-field systems.

\section{Sequence 6: Sagittal, transverse, and coronal $T_{1}$-weighted imaging}

1. Perform sagittal, transverse, and coronal $T_{1}$-weighted scan (sequence 6; Table A7.5.7).

Since the pathology may be very small (a few millimeters in dimension) it is advantageous to acquire all three imaging orientation planes (Table A7.5.7). Because of partial volume averaging, it may not be possible to see a small choroidal lesion on more than one slice, and it is frequently impossible to predict which plane will be best for visualization. 
Table A7.5.5 Parameters for Transverse $T_{1}$-Weighted Images (Sequence 4)

Patient position

Scan type

Imaging plane (orientation)

Central slice or volume center

Echo time $\left(T_{\mathrm{E}}\right)$

Repeat time $\left(T_{\mathrm{R}}\right)$

Flip angle (FA)

Fields of view $\left(\mathrm{FOV}_{\mathrm{x}}, \mathrm{FOV}_{\mathrm{y}}\right)$

Resolution $(\Delta x, \Delta y)$

Number of data points collected $\left(N_{\mathrm{x}}, N_{\mathrm{y}}\right)$

Display matrix $\left(D_{\mathrm{x}}, D_{\mathrm{y}}\right)$

Slice thickness $(\Delta z)$

Number of slices

Slice gap

Number of acquisitions $\left(N_{\text {acq }}\right)$

Swap read and phase encoding

Saturation pulses

Contrast enhancement

Scan time
Supine

Spin echo

Transverse

Laser light centered on nose

As short as possible

$500 \mathrm{msec}$

$90^{\circ}$

$240 \mathrm{~mm}, 240 \mathrm{~mm}$

$0.94 \mathrm{~mm}, 0.94 \mathrm{~mm}$

256,256

256,256

$3-5 \mathrm{~mm}$

20

$1-2 \mathrm{~mm}$

$1-2$

Yes

Fat saturation

Yes

2-4 min

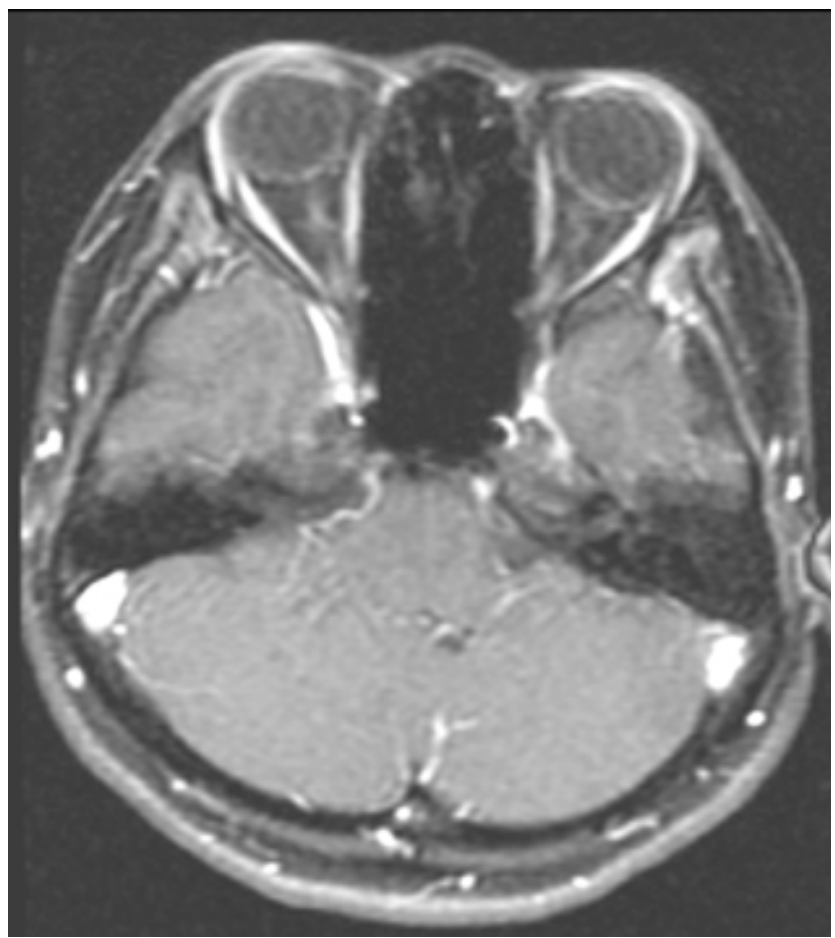

Figure A7.5.4 Transverse $T_{1}$-weighted contrast enhanced image of the head with fat suppression. Note the intense normal contrast enhancement of the extraocular muscles and the lack of signal from the orbital fat which allows for evaluation of the orbital contents. The suppression of the fat increases the signal intensity differences between the contrast enhanced structures and the other orbital structures, particularly the optic nerve. Without fat saturation the contrast enhancement is "lost" in the fat signal. Imaging the entire head also allows for the evaluation of intracranial structures, which may account for vision changes (occipital lobe lesions). 


\section{Sequence 7: Spin-echo $T_{2}$-weighted sequence}

2. Perform spin-echo $T_{2}$-weighted scan (sequence 7; Table A7.5.8).

The imaging orientation plane that best demonstrates the lesion on the $T_{1}$-weighted images is also used for the $T_{2}$-weighted images (Table A7.5.8). When evaluating choroidal masses, it is important to characterize the relaxation properties of the mass, since melanomas have odd signal characteristics that are frequently quite different from other tumors: they have much shorter $T_{1}$ relaxation times as a result of the paramagnetic properties of melanin. Documenting odd signal behavior can help to differentiate a melanoma from another

A

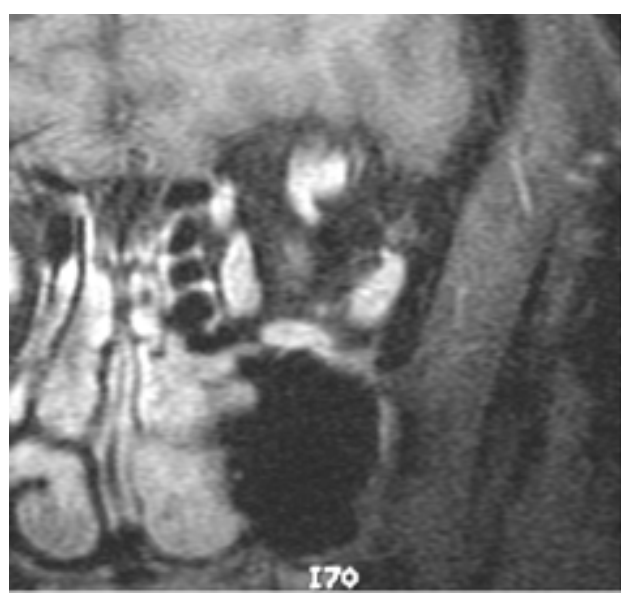

B

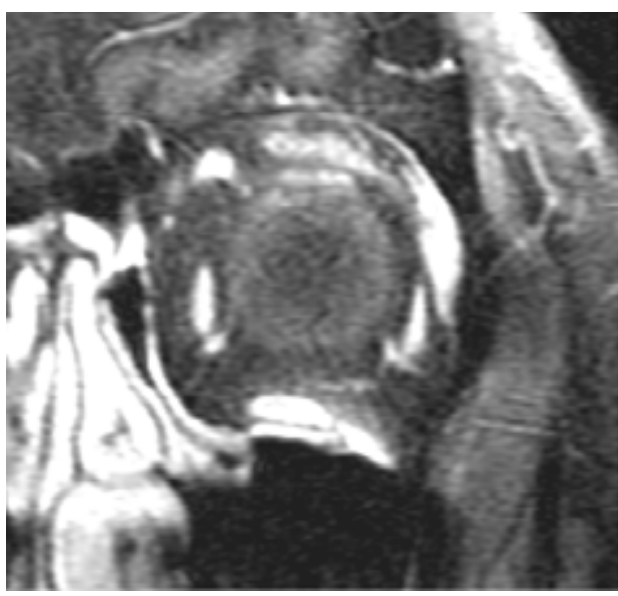

Figure A7.5.5 Coronal $T_{1}$-weighted fat saturation images obtained with a surface coil. Image $(\mathbf{A})$ is centered just behind the globe. Note the lack of enhancement of the optic nerve, in contrast to the extraocular muscles. Image (B) is centered through the globe and allows detailed imaging of the globe. Note the intense enhancement of the nasal cavity mucosa.

Table A7.5.6 Parameters for Coronal $T_{1}$-Weighted Contrast Enhanced Images with Fat Saturation (Sequence 5)

\begin{tabular}{ll}
\hline Patient position & Supine \\
Scan type & Spin echo \\
Imaging plane (orientation) & Coronal \\
Central slice or volume center & Laser light centered on nose \\
Echo time $\left(T_{\mathrm{E}}\right)$ & As short as possible \\
Repeat time $\left(T_{\mathrm{R}}\right)$ & $500 \mathrm{msec}$ \\
Flip angle $(\mathrm{FA})$ & $90^{\circ}$ \\
Fields of view $\left(\mathrm{FOV}_{\mathrm{x}}, \mathrm{FOV}_{\mathrm{y}}\right)$ & $240 \mathrm{~mm}, 240 \mathrm{~mm}$ \\
Resolution $(\Delta x, \Delta y)$ & $0.94 \mathrm{~mm}, 0.94 \mathrm{~mm}$ \\
Number of data points collected $\left(\mathrm{N}_{\mathrm{x}}, \mathrm{N}_{\mathrm{y}}\right)$ & 256,256 \\
Display matrix $\left(D_{\mathrm{x}}, D_{\mathrm{y}}\right)$ & 256,256 \\
Slice thickness $(\Delta z)$ & $3-5 \mathrm{~mm}$ \\
Number of slices & 20 \\
Slice gap & $1-2 \mathrm{~mm}$ \\
Number of acquisitions $\left(N_{\mathrm{acq}}\right)$ & $1-2$ \\
Swap read and phase encoding & No \\
Saturation pulses & Fat saturation \\
Contrast enhancement & Yes \\
Scan time & $2-4$ min \\
\hline
\end{tabular}


tumor, such as a lymphoma, metastases, or a hemangioma. It is possible to assess the $T_{2}$ properties of the pathology more accurately with a four-echo train than with one or two $T_{E}$ images alone.

\section{Sequence 8: $T_{1}$-weighted contrast-enhanced sequence}

3. Perform $T_{1}$-weighted contrast-enhanced scan (sequence 8; Table A7.5.9).

Contrast-enhanced high-resolution surface coil imaging (Figs. A7.5.4 and A7.5.5) can help to characterize small lesions and potentially make them more conspicuous (Table A7.5.9).

Table A7.5.7 Parameters for Sagittal, Transverse, anad Coronal $T_{1}$-Weighted Imaging (Sequence 6)

\begin{tabular}{ll}
\hline Patient position & Supine \\
Scan type & Spin echo \\
Imaging plane (orientation) & Sagittal, transverse, and coronal \\
Central slice or volume center & Laser light centered on nose \\
Echo time $\left(T_{\mathrm{E}}\right)$ & As short as possible \\
Repeat time $\left(T_{\mathrm{R}}\right)$ & $500 \mathrm{msec}$ \\
Flip angle $(\mathrm{FA})$ & $90^{\circ}$ \\
Fields of view $\left(\mathrm{FOV}_{\mathrm{x}}, \mathrm{FOV}_{\mathrm{y}}\right)$ & $240 \mathrm{~mm}, 240 \mathrm{~mm}$ \\
Resolution $(\Delta x, \Delta y)$ & $0.94 \mathrm{~mm}, 0.94 \mathrm{~mm}$ \\
Number of data points collected $\left(N_{\mathrm{x}}, N_{\mathrm{y}}\right)$ & 256,256 \\
Display matrix $\left(\mathrm{D}_{\mathrm{x}}, \mathrm{D}_{\mathrm{y}}\right)$ & 256,256 \\
Slice thickness $(\Delta z)$ & $3 \mathrm{~mm}$ \\
Number of slices & 20 \\
Slice gap & $1-2 \mathrm{~mm}$ \\
Number of acquisitions $\left(N_{\mathrm{acq}}\right)$ & $1-2$ \\
Swap read and phase encoding & Swap if transverse plane is used \\
Saturation pulses & Not applicable \\
Scan time & $\sim 2-4$ min \\
\hline
\end{tabular}

Table A7.5.8 Parameters for $T_{2}$-Weighted Image in the Plane Best Demonstrating the Pathology (Sequence 7)

Patient position

Scan type

Imaging plane (orientation)

Central slice or volume center

Echo time $\left(T_{\mathrm{E}}\right)$

Repeat time $\left(T_{\mathrm{R}}\right)$

Flip angle (FA)

Fields of view $\left(\mathrm{FOV}_{\mathrm{x}}, \mathrm{FOV}_{\mathrm{y}}\right)$

Resolution $(\Delta x, \Delta y)$

Number of data points collected $\left(N_{\mathrm{x}}, N_{\mathrm{y}}\right)$

Display matrix $\left(\mathrm{D}_{\mathrm{x}}, \mathrm{D}_{\mathrm{y}}\right)$

Slice thickness $(\Delta z)$

Number of slices

Slice gap

Number of acquisitions $\left(N_{\text {acq }}\right)$

Swap read and phase encoding

Saturation pulses

Scan time
Supine

Spin echo

Best plane from the result of sequence 6 Laser light centered on nose $30,60,90,120 \mathrm{msec}$ $5000 \mathrm{msec}$ $90^{\circ}$

$240 \mathrm{~mm}, 240 \mathrm{~mm}$

$0.94 \mathrm{~mm}, 0.94 \mathrm{~mm}$

256, 256

256, 256

$3 \mathrm{~mm}$

20

$1 \mathrm{~mm}$

1-2

Swap if transverse plane is used Not applicable

$\sim 2 \min$ 
Table A7.5.9 Parameters for $T_{1}$-Weighted Contrast Enhanced Images (Sequence 8)

$\begin{array}{ll}\text { Patient position } & \text { Supine } \\ \text { Scan type } & \text { Spin echo } \\ \text { Imaging plane (orientation) } & \text { Best plane from prior exams } \\ \text { Central slice or volume centr } & \text { Laser light centered on nose } \\ \text { Echo time }\left(T_{\mathrm{E}}\right) & \text { As short as possible } \\ \text { Repeat time }\left(T_{\mathrm{R}}\right) & 500 \mathrm{msec} \\ \text { Flip angle }(\mathrm{FA}) & 90^{\circ} \\ \text { Fields of view }\left(\mathrm{FOV}_{\mathrm{x}}, \mathrm{FOV}_{\mathrm{y}}\right) & 240 \mathrm{~mm}, 240 \mathrm{~mm} \\ \text { Resolution }(\Delta x, \Delta y) & 0.94 \mathrm{~mm}, 0.94 \mathrm{~mm} \\ \text { Number of data points collected }\left(N_{\mathrm{x}}, N_{\mathrm{y}}\right) & 256,256 \\ \text { Display matrix }\left(D_{\mathrm{x}}, D_{\mathrm{y}}\right) & 256,256 \\ \text { Slice thickness }(\Delta z) & 3 \mathrm{~mm} \\ \text { Number of slices } & 20 \\ \text { Slice gap } & 1 \mathrm{~mm} \\ \text { Number of acquisitions }\left(N_{\mathrm{acq}}\right) & 1 \\ \text { Swap read and phase encoding } & \text { Swap if transverse plane is used } \\ \text { Saturation pulses } & \text { If desired } \\ \text { Contrast enhancement } & \text { Yes } \\ \text { Scan time } & \sim 2-4 \text { min }\end{array}$

Table A7.5.10 Parameters for STIR-Weighted Contrast Enhanced Images (Sequence 9)

Patient position

Scan type

Imaging plane (orientation)

Central slice or volume center

Echo time $\left(T_{\mathrm{E}}\right)$

Repeat time $\left(T_{\mathrm{R}}\right)$

Inversion time $\left(T_{\mathrm{I}}\right)$

Flip angle (FA)

Fields of view $\left(\mathrm{FOV}_{\mathrm{x}}, \mathrm{FOV}_{\mathrm{y}}\right)$

Resolution $(\Delta x, \Delta y)$

Number of data points collected $\left(N_{\mathrm{x}}, N_{\mathrm{y}}\right)$

Display matrix $\left(D_{\mathrm{x}}, D_{\mathrm{y}}\right)$

Slice thickness $(\Delta z)$

Number of slices

Slice gap

Number of acquisitions $\left(N_{\text {acq }}\right)$

Swap read and phase encoding

Saturation pulses

Contrast enhancement

Scan time
Supine

Short tau inversion recovery, spin echo

Best plane from prior exams

Laser light centered on nose

As short as possible

$1600 \mathrm{msec}$

$90 \mathrm{msec}$

$180^{\circ}$

$240 \mathrm{~mm}, 240 \mathrm{~mm}$

$0.94 \mathrm{~mm}, 0.94 \mathrm{~mm}$

256,256

256,256

$3 \mathrm{~mm}$

20

$1 \mathrm{~mm}$

1

Swap if transverse plane is used

If desired

Yes

$\sim 7 \mathrm{~min}$ 
Fat saturation can be added, but will have the usual limitations. Motion of the globe and blood flow-related artifacts become more troublesome with these types of sequences.

\section{Sequence 9: Short tau inversion recovery (STIR) weighted contrast enhanced sequence}

4. Perform Short tau inversion recovery (STIR) weighted contrast enhanced scan (sequence 9; Table A7.5.10).

On systems that do not have fat saturation, short tau inversion recovery (STIR) sequences can be of value (Table A7.5.10). This is true for lower-field magnets in particular. This sequence suppresses the fat by choosing an inversion time that brings the signal to fat near null (signal void). This technique is not specific for fat though, but rather for suppression of a range of short $T_{1}$ tissues. In this sequence, the fat will have a lower signal than usual, enhancing the contrast difference with other tissues.

\section{COMMENTARY}

\section{Background Information}

Orbital pathology can be very diverse, so it is difficult to predict which technique will be ideal for any one patient. Pathology embedded within the orbital fat is frequently easier to evaluate, since the fat acts as a natural contrast agent and outlines the pathology (which frequently has lower signal and longer $T_{1}$ relaxation times), though this may not be true for orbital lipomas. Lipomas usually occur in the anterior portion of the orbit so it is easier to recognize the pathology. Thyroid ophthalmopathy is frequently associated with infiltration of the fat without much of a change in signal properties, in which case one sees proptosis without an apparent mass. One can usually differentiate hemorrhage in the orbit from fat by its signal characteristics on $T_{2}$ or gradient echo imaging. Iron products in the form of met-hemoglobin can have high signal on $T_{1^{-}}$ weighted imaging, but frequently demonstrate high signal properties on $T_{2}$-weighted images as well. More acute or chronic hemorrhage (hemosiderin) can demonstrate very low signal due to the marked $T_{2}$ shortening that is associated with these strongly magnetic components.

Usually, the orbital fat clearly outlines pathology of the extraocular muscles. One can see infiltration of the muscles without enlargement on noncontrast $T_{2}$-weighted or contrast-enhanced $T_{1}$-weighted imaging.

Pathology of the paranasal sinuses is usually obvious since the air spaces of the sinuses should be seen as signal voids. Inspissated secretions may mimic the appearance of air spaces, with no signal visible. This appearance will be contradictory to findings seen on CT or plain radiographs.

Evaluation of the optic nerve is very important. Therefore, contrast-enhanced sequences are important, since pathology may be present even when the nerve's normal configuration is unaltered, as in the case of optic neuritis. Sequences should evaluate the intracranial segments of the optic nerves, cranial nerves, cerebrospinal fluid (CSF) spaces (optic nerve sheath, Meckel's cave, prepontine cistern, and the suprasellar cistern) and the vascular spaces (cavernous sinus, carotid artery). Pathology frequently bridges these structures, as in the case of perineural spread of tumor.

Despite its relatively simple configuration, the globe is difficult to image because of the small size of its components and its tendency to move. Surface coil and fat saturation imaging may be helpful.

\section{Critical Parameters and Troubleshooting}

Suppression of globe motion is almost impossible, which is unfortunate, since it can severely mar the quality of an exam, particularly if the exam is focused on the globe. Fast image sequences may help suppress the effects of motion, but these sequences frequently have lower resolution and simply do not have good enough image quality to answer some clinical questions.

If the patient has metallic hardware, fat saturation imaging may be impossible. In fact, routine imaging of the orbit may be impossible in some patients who have dental hardware, and even patients with no dental hardware can have bulk magnetic susceptibility artifacts. Magnetic susceptibility pads can be placed over the eyes to limit artifacts.

The magnetic field within a patient is not constant; it varies with the composition of the tissue being studied. The susceptibility difference between air and soft tissues leads to an 
inhomogeneous field across the tissue. Therefore, when fat saturation is utilized, there may be regions in the tissue that are accidentally suppressed and parts of the fat that are not. In this case, there may be an appearance of false enhancement. If there is question about the true source of the signal, it is advisable to acquire images without saturation pulses.

Chemical shift artifacts can obscure detail, particularly at the margins of the extraocular muscles and the globe. The best way to avoid this problem is to use fat saturation techniques.

\section{Anticipated Results}

The goal of the exam is to identify and characterize all of the orbital structures and their adjacent connections. Since these structures can have a wide variation in their signal properties, it is necessary to use multiple imaging sequences. It should be possible to follow the optic nerve from the globe to the optic radiations. Each extraocular muscle should be identifiable and the lacrimal glands should be both visible and symmetric. The fatty spaces of the pterygopalatine fissure, superior orbital fissure, and inferior orbital fissure should be visible. In addition, the superior orbital fissure, foramen rotundum, and optic canal should be well demonstrated, including their associated nerves and vessels. It is also important to exclude pathology of the cavernous sinus and carotid arteries.

If there is pathology, its complete extent should be fully evaluated. In the case of the orbit, it is possible that pathology may track all the way back to the occipital poles. Since the superior inferior extension of pathology can also be very broad, it may also be important to image all the way back to the foramen magnum.

\section{Acknowledgements}

The authors would like to thank Linda Chakeres for the editing of the manuscript.

\section{Literature Cited}

Amano, Y., Amano, M., and Kumazaki, T. 1997. Normal contrast enhancement of extraocular muscles: Fat-suppressed MR findings. AJNR Am. J. Neuroradiol. 18:161-164.
Anzai, Y., Lufkin, R.B., Jabour, B.A., and Hanafee, W.N. 1992. Fat-suppression failure simulating pathology on frequency-selective fat-suppression images of the head and neck. AJNR Am.J. Neuroradiol. 13(3):879-884.

Atlas, S.W., Bilaniuk, L.T., Zimmerman, R.A., et al. 1987. Orbit: Initial experience with surface coil spin-echo imaging at 1.5 T. Radiology 164:501509.

Barakos, J.A., Dillon, W.P., and Chew, W.M. 1991. Orbit, skull base and pharynx: Contrast enhanced fat suppression MR imaging. Radiology 179:191-198.

Herrick, R.C., Hayman, L.A., Taber, K.H., et al. 1997. Artifacts and pitfalls in MR imaging of the orbit: A clinical review. Radiographics 17(3):707-724.

Kelly, W.M., Paglen, P.G., Pearson, J.A., et al. 1986. Ferromagnetism of intraocular foreign body causes unilateral blindness after MR study. AJNR Am. J. Neuroradiol. 7:243-245.

Lagouros, P.A., Langer, B.G., Peyman, G.A., et al. 1987. Magnetic resonance imaging and intraocular foreign bodies. Arch. Ophthalmol. 105:551-553.

Mafee, M.F., Ainbinder, D., Afshani, E., and Mafee, R. 1996. The eye, imaging of the globe, orbit, and visual pathways. Neuroimaging Clin. North Am. 6:29-59.

Otto, P.M., Otto, R.A., Virapongse, C., et al. 1992. Screening test for detection of metallic foreign objects in the orbit before magnetic resonance imaging. Invest Radiol 27:308-311.

Sacco, D.C., Steiger, D.A., Bellon, E.M., et al. 1987. Artifacts caused by cosmetics in MR imaging of the head. AJR Am. J. Roentgenol. 148:10011004.

Shellock, F.G. 1996. Pocket Guide to MR Procedures and Metallic Objects. Lippincott-Raven, Philadelphia.

\section{Key References}

Mafee et al., 1996. See above.

This reference is a general review article focused on imaging of the orbit and visual system.

Contributed by Donald William Chakeres and Eric C. Bourekas

Ohio State University College of Medicine and Public Health

Columbus, Ohio 